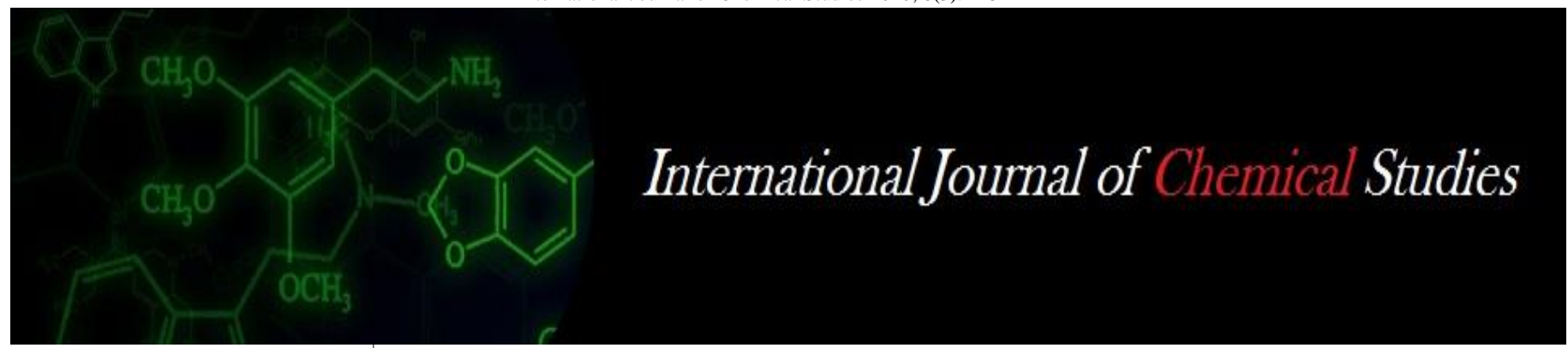

P-ISSN: 2349-8528

E-ISSN: 2321-4902

www.chemijournal.com

IJCS 2020; 8(3): 2134-2141

(C) 2020 IJCS

Received: 16-03-2020

Accepted: 20-04-2020

Sowjanya BA

Department of Genetic and Plant Breeding, University of

Agricultural Sciences, Dharwad,

Karnataka, India

Sridevi 0

Department of Genetic and Plant

Breeding, University of

Agricultural Sciences, Dharwad,

Karnataka, India
Corresponding Author: Sowjanya BA

Department of Genetic and Plant Breeding, University of

Agricultural Sciences, Dharwad,

Karnataka, India

\section{Combining ability and heterosis studies in tomato (Solanum lycopersicum L.) under ToLCV disease stress condition}

\section{Sowjanya BA and Sridevi O}

DOI: https://doi.org/10.22271/chemi.2020.v8.i3ad.9526

\begin{abstract}
The present investigation was carried out by crossing 15 lines (susceptible to ToLCV) with 3 testers (resistant to ToLCV) in line $\times$ tester design, the resultant 45 hybrids along with parents were evaluated for combining ability at Botany Garden, Department of Genetics and Plant Breeding, College of Agriculture, Dharwad during Rabi, 2018-19 and also screened for ToLCV resistant. Analysis of variance revealed highly significant differences among all the $F_{1}$ hybrid means and their respective six parental values for all examined traits and revealed the predominance of non-additive gene action for all the traits. In respect of both gca and sca effects, the parents and hybrids differed significantly. Among the parents, L4, L5, L6, L7 and AVTO-1219 were the best general combiners for yield per plant and other characters under study, and these may be used as valuable donors in the hybridization programme. Among the crosses, $\mathrm{L} 3 \times \mathrm{T} 3, \mathrm{~L} 8 \times \mathrm{T} 1, \mathrm{~L} 13 \times \mathrm{T} 3$ and $\mathrm{L} 14 \times \mathrm{T} 2$ were the most valuable combiners for yield per plant and other characters under study could be utilized for heterosis breeding programmes. The crosses L $3 \times$ T3, L1 $\times$ T3 and L7 $\times$ T3 exhibited superior mid parent heterosis for yield per plant. Out of 45 hybrids evaluated for performance in leaf curl stress condition under natural condition, five hybrids L6 $\times$ T2, L7 $\times \mathrm{T} 3, \mathrm{~L} 10 \times \mathrm{T} 2, \mathrm{~L} 12 \times \mathrm{T} 3$ and $\mathrm{L} 13 \times \mathrm{T} 3$ exhibited moderate resistance reaction.
\end{abstract}

Keywords: Tomato, gene action, combining ability, heterosis, ToLCV

\section{Introduction}

Among the vegetable crops, tomato (Solanum lycopersicum L.) is one of the most popular and widely grown vegetable crop and is native of Peru, South America (Rick, 1969) ${ }^{[21]}$. It belongs to the family Solanaceae with chromosome number $2 n=2 x=24$. It is mainly consumed as salad, juice, sauce, ketchup and whole canned fruit. It is rich source of antioxidants, vitamin A, vitamin $\mathrm{C}$ and minerals like $\mathrm{Ca}, \mathrm{P}$ and $\mathrm{Fe}$ in diet. Tomato is grown majorly in tropical and subtropical areas. Because of its day neutral, self-pollinated nature it can be grown throughout the year.

Worldwide growth and spread of tomato as a vegetable crop is limited by the fact that it is affected by a number of diseases causing substantial yield loss and also affecting the quality of fruits. Besides fungal, bacterial and mycoplasmal infection, it is also affected by large number of viral diseases. Among the various diseases, in India tomato leaf curl virus (ToLCV) is a major viral disease which cause 90 to 100 per cent yield loss in the affected areas. In southern India it affects during summer season and in northern India in autumun season (Banerjee and Kalloo, 1987a) ${ }^{[5]}$.

In the face of mounting pressure, sustainable advance in tomato productivity and production is perhaps salutary to realize nutritional security particularly when India is facing demographic watershed. To meet the ever-increasing demand for this vegetable, there is a need to develop superior, stable and resistant varieties and also hybrids with better yield and quality.

By the application of biometrical principles, genetic architecture of yield can be better understood. Several biometrical methods provide the information on the combining ability status of parental lines. One of the technique widely used is line $\times$ tester analysis developed by Kempthrone (1957) ${ }^{[14]}$. This method provides reliable information on magnitude of additive and non-additive components of the lines based on the general combining ability (GCA) effects of parents and their hybrid combinations. This helps to assess the nature of gene action and in identifying superior parental lines for their per se performance. 
The most excellent combinations with high GCA of individual lines are useful to get more desirable recombination which enables for further improvement of crop.

Heterosis breeding is one of the method to improve the yield and quality. The importance of heterosis breeding has been recognized widely in most of the vegetable crops. Hedrick and Booth (1968) ${ }^{[11]}$ were the first to observe heterosis in tomato for superior yield and for higher number of fruits per plant. Choudhary et al. (1965) ${ }^{[8]}$ emphasized the extensive exploitation of heterosis to maximize tomato production. Manifestation of heterosis in tomato is in the form of higher vigour, faster growth and development, increased productivity, earliness in maturity, higher levels of resistance to biotic and abiotic stresses (Yordanov, 1983) ${ }^{[32]}$. The study of heterosis provides the basis for the utilization of valuable hybrid combinations in the breeding.

Development of hybrids is important to increase yield per se and enhance resistance for most of the diseases. Choosing of suitable parents is very important in any hybrid breeding programme to realize significant heterosis for the economic traits.

\section{Materials and Methods}

The present study was carried out at Botany garden, University of Agricultural Sciences, Dharwad during rabi season 2018. The study used $F_{1}$ population developed by crossing 15 lines (susceptible to ToLCV) of tomato which were developed in the University of Agricultural Sciences, Dharwad Department of Genetics and Plant Breeding with 3 testers (CLN2768A, CLN777H and AVTO-1219) which were obtained from AVRDC Taiwan resistant to ToLCV disease. Forty five crosses were made manually by hand emasculation and pollination. The experiment was laid out by following Randomized Complete Block Design (RCBD). The experiment was replicated two times. The crop grown according to the standard cultural recommendations for the area and thirty days old seedlings of the plants were transplanted into the experimental plot with a spacing of $60 \times$ $60 \mathrm{~cm}$.
The plant growth measurements include days to 50 per cent flowering (DFF), plant height $(\mathrm{PH})$, number branches per plant (NBPP), number of cluster per plant (NCPP), number of fruits per cluster (NFPC), number of fruits per plant (NFPP), polar length of fruit (PLF), equatorial length of fruit (ELF), fruit shape index (FSI), pericarp thickness of fruit (PTF), number of locules per fruit (NLPF), total soluble solids (TSS), average fruit weight (AFW) and yield per plant (YPP).

\section{Screening of $F_{1}$ 's for ToLCV disease resistance}

All forty five hybrids developed by crossing 15 lines with 3 testers along with parents were naturally screened for ToLCV disease resistance in summer 2019 by transplanting twenty five days old artificially inoculated seedlings to field condition and Plants were examined visually for disease symptoms at 30, 60 and 90 days after transplanting and observations recorded.

\section{Per cent ToLCV disease incidence}

The incidence of ToLCV was recorded at 30, 45 and 60 days after transplanting. The number of plants infected in each entry was recorded and computed by using the following formula.

$$
\text { Per cent disease incidence }(\%)=\frac{\text { Total number of plants infected with ToLCV }}{\text { Total number of plants }} \times 100
$$

\subsubsection{ToLCV disease symptom severity}

An arbitrary scale was employed for scoring the disease severity as described by Lapidot and Friedmann (2002) (Table 1).

\section{Data analysis}

Data were recorded on five randomly selected plants from each replication of parents, hybrids and two checks and analyzed by analysis of variance (ANOVA) at 5\% levels of significance. Combining ability analysis was done using line $\times$ tester method (Kempthorne, 1957) ${ }^{[14]}$. The heterosis $(\%)$ over better parent by using the formula proposed by Falconer $(1981)^{[9]}$.

Table 1: ToLCV disease symptom severity scale used for screening the disease in tomato

\begin{tabular}{|c|c|c|c|c|}
\hline Class & Category & Incidence & Score & Symptom severity \\
\hline Resistant & R & 0 & 0 & No symptom \\
\hline Moderately Resistant & MR & Upto 25\% & 1 & Light yellowing along the leaf margins and mild vein clearing \\
\hline Tolerant & $\mathrm{T}$ & $26-50 \%$ & 2 & Yellowing of leaves and slight curling, growth, flowering and yield not greatly \\
affected.
\end{tabular}

\section{Results and Discussion}

Data in table 2 reflected that the analysis of variances due to genotypes and its components (parents, crosses and parents vs. crosses) were highly significant for all the traits studied except number of fruits per cluster. These results indicated wide between the parental materials used in this study. It also indicated that the variance due to lines was highly significant for all the traits studied except for fruits per cluster and fruit shape index and variance due to testers were significant for all the traits except for days to 50 per cent flowering, number of clusters per plant, number of fruits per cluster, equatorial length of fruit, fruit shape index, fruit shape index, pericarp thickness and number of locules. The variances due to line $x$ tester interactions, representing specific combining ability, were also highly significant for all the traits, which suggested manifestation of parental genetic variability in their crosses. In present study, gca variances was found to be significant for all the traits studied except number of fruits per cluster, number of fruits per plant, fruit shape index, number of locules per fruit and TSS and sca variance was found to be significant for all the traits studied except days to 50 per cent flowering (Table 2). This suggested that both additive and non-additive variances were important in the inheritance of these characters. Significant of both variances have been reported by Singh et al. (2010) ${ }^{[28]}$ for days to $50 \%$ flowering; Asati et al. (2007) ${ }^{[4]}$ and Singh et al. (2010) ${ }^{[28]}$ for plant height at final harvest; Premalakshme et al. (2006) ${ }^{[20]}$, Singh et al. (2010) ${ }^{[28]}$ and Asati et al. (2007) ${ }^{[4]}$ for number of fruits 
per plant, Singh et al. (2008) [27] for average fruit weight; Bhatt et al. (2000) ${ }^{[6]}$ and Singh et al. (2010) ${ }^{[28]}$ for fruit yield per plant; Himanshu et al. (2008) ${ }^{[12]}$ for pericarp thickness; Singh et al. (2005) ${ }^{[26]}$ and Joshi and Kohli (2006) ${ }^{[13]}$ for total soluble solids;

The ratio of GCA to SCA variance was less than unity for all the characters including fruit yield per plant indicating predominance of non-additive gene action and hence, improvement in the yield can be achieved through heterosis breeding program (Table 3). These findings are in agreement with Ahmad et al. (2009) [2], Farzane et al. (2012) ${ }^{[10]}$ and Mali and Patel (2014) ${ }^{[17]}$ for plant height at final harvest; Angadi et al. (2012) [3] for number of clusters per plant; Angadi et al. (2012) ${ }^{[3]}$ and Yadav et al. (2013) ${ }^{[30]}$ for number of fruits per plant; Yadav et al. (2013) [30], Saleem et al. (2013) [22] and Agarwal et al. (2014) [1] for average fruit weight;

Nature and magnitude of combining ability effects provide guidelines for identifying parents and their utilization in hybridization programme. In the present study, significant gca effects were observed for most of the characters (Table 4). Among the parents L1, L5, L6, L7, L9, L10, L15 and AVTO1219 (T3) were good general combiner for fruit yield per plant and some of its direct components. The females viz., L2, L4, L8, L13 and L14 were found to be poor general combiner for fruit yield as well as yield attributing characters. Among males, CLN2777H (T2) was found to be poor general combiner for most of the traits followed by CLN2768A (T1). The estimates of $g c a$ effect further revealed that the parental lines showing high $\mathrm{gca}$ effects for fruit yield per plant also exhibited high to average $g c a$ effects for one or more yield components. Among parents, high gca effect for average fruit weight was found in L4, L5, L6, L7, L9, L10, L13 and AVTO-1219 (T3) which associated with positive and significant gca effects for average fruit weight. Almost identical result have been reported by Himanshu et al. (2008) [12], Ahmad et al. (2009) [2], Farzane et al. (2012) [10], Narasimhamurthy and Gowda (2013) ${ }^{[18]}$.

Specific combining ability effects (SCA) is the manifestation of non-additive component of genetic variance and associated with interaction effects, which may be due to dominance and epistatic component of genetic variation that are non-fixable in nature. Such non-fixable components are potential parameters for heterosis breeding which is very much useful in tomato where commercial exploitation of heterosis is feasible. The estimation of sca effects (Table 5) show that, the crosses L3 $\times$ T3, L8 $\times \mathrm{T} 1, \mathrm{~L} 13 \times \mathrm{T} 3$ and L14 $\times \mathrm{T} 2$ had positive and significant values for yield per plant. The crosses $\mathrm{L} 2 \times \mathrm{T} 1, \mathrm{~L} 10 \times \mathrm{T} 3$ and L1 $\times \mathrm{T} 3$ exhibited positive and highly significant values for average fruit weight. The crosses L3 $\times$ T3, L8 $\times$ T1 and L14 $\times$ T1 had positive and significant or highly significant values for number of fruits per plant.

\section{Heterosis}

Significant efforts have been made for exploitation of heterosis in different yield contributing traits to find the feasible cross for the production of $\mathrm{F}_{1}$ hybrids. The hybrids showing high heterosis have good chances to identify desirable lines in succeeding generations as compared to hybrids having low heterotic effects (Sharif et al., 2001) ${ }^{[24]}$. All the crosses exhibited significant mid parent heterosis in majority of the traits indicating a predominance of nonadditive gene action in the genetic control of these traits. The highest mid parent heterosis were exhibited by the hybrids viz $\mathrm{L} 4 \times \mathrm{T} 3$ for plant height, $\mathrm{L} 10 \times \mathrm{T} 2, \mathrm{~L} 3 \times \mathrm{T} 1$ and $\mathrm{L} 6 \times \mathrm{T} 2$ for the trait number of branches per plant, L12 $\times \mathrm{T} 3, \mathrm{~L} 10 \times \mathrm{T} 2$ and L6 $\times$ T2 hybrids exhibited for the trait number of clusters per plant. Highest mid parent heterosis for number of fruits per cluster hybrids exhibited by the crosses L5 $\times$ T1, L14 $\times$ $\mathrm{T} 1, \mathrm{~L} 3 \times \mathrm{T} 3$ and $\mathrm{L} 4 \times \mathrm{T} 2$. The crosses $\mathrm{L} 2 \times \mathrm{T} 1, \mathrm{~L} 2 \times \mathrm{T} 3, \mathrm{~L} 2$ $\times \mathrm{T} 2$ and L4 $\times$ T2 exhibited highest mid-parent value for the trait number of fruits per plant. Hybrids L1 $\times \mathrm{T} 3, \mathrm{~L} 4 \times \mathrm{T} 3$, $\mathrm{L} 13 \times \mathrm{T} 2, \mathrm{~L} 13 \times \mathrm{T} 3, \mathrm{~L} 10 \times \mathrm{T} 3$ and L6 $\times \mathrm{T} 3$ exhibited highest mid parent heterosis for the trait average fruit weight. Highest mid parent heterosis for the trait polar length of fruit observed in the crosses $\mathrm{L} 1 \times \mathrm{T} 3, \mathrm{~L} 4 \times \mathrm{T} 3$ and L6 $\times$. For th trait equatorial length of the fruit highest mid parent heterosis observed in the hybrids L1 $\times$ T3, L4 $\times$ T3 and L6× T3. The crosses $\mathrm{L} 3 \times \mathrm{T} 3$ and $\mathrm{L} 2 \times \mathrm{T} 3$ exhibited highest mid parent heterosis for the trait fruit shape index. For pericarp thickness the crosses $\mathrm{L} 13 \times \mathrm{T} 3, \mathrm{~L} 15 \times \mathrm{T} 2$ and $\mathrm{L} 1 \times \mathrm{T} 3$ recorded highest significant value over mid-parent heterosis. The crosses L $1 \times$ $\mathrm{T} 3, \mathrm{~L} 11 \times \mathrm{T} 2, \mathrm{~L} 13 \times \mathrm{T} 2$ and L15 $\times \mathrm{T} 2$ exhibited highest mid parent heterosis for the trait number of locules per fruit. Highest mid parent heterosis for TSS exhibited by the crosses $\mathrm{L} 3 \times \mathrm{T} 1, \mathrm{~L} 10 \times \mathrm{T} 1, \mathrm{~L} 3 \times \mathrm{T} 2, \mathrm{~L} 14 \times \mathrm{T} 2, \mathrm{~L} 1 \times \mathrm{T} 1$ and $\mathrm{L} 13 \times$ T1. Highest mid parent heterosis for yield per plant obtained in the cross combination $\mathrm{L} 4 \times \mathrm{T} 3, \mathrm{~L} 5 \times \mathrm{T} 2, \mathrm{~L} 7 \times \mathrm{T} 2, \mathrm{~L} 4 \times \mathrm{T} 2$ and L1 $\times$ T1 . Assuming that epistasis is absent, the cause of heterosis can only be attributed to the dominant gene action. The results were in accordance with the findings of Makesh $e t$ al. (2003) ${ }^{[16]}$, Bhatt et al. (2004) ${ }^{[7]}$, Tiwari and Lal (2004) ${ }^{[29]}$, Nitu et al. (2010) ${ }^{[19]}$, Singh and Asati (2011) ${ }^{[25]}$ and Shankar et al. (2013) ${ }^{[23]}$.

\section{Screening of hybrids for ToLCV disease resistance}

The hybrids were evaluated based on 0 to 5 disease rating scale. The reaction of hybrids is presented in Table 1 and 2 . Among 45 hybrids, five hybrids L6 $\times$ T2, L7 × T3, L10 × T2, L12 $\times$ T3 and L13 $\times$ T3 were moderately resistant, twentythree were tolerant and remaining 15 were susceptible to ToLCV disease. Among 15 lines two lines 1602-15 and 160250 were tolerant, three lines 1601-36, 1602-03 and 1602-21 were highly susceptible remaining ten lines were susceptible and all the testers were resistant.

Table 2: Analysis of variance (ANOVA) for combining ability for yield and yield component traits in tomato

\begin{tabular}{|c|c|c|c|c|c|c|c|c|}
\hline $\begin{array}{l}\text { Source of } \\
\text { Variations }\end{array}$ & $\begin{array}{c}\text { Degrees } \\
\text { of } \\
\text { freedom }\end{array}$ & $\begin{array}{c}\text { Days to } 50 \% \\
\text { flowering }\end{array}$ & $\begin{array}{c}\text { Plant height } \\
\text { (cm) }\end{array}$ & $\begin{array}{c}\text { Number of } \\
\text { branches per } \\
\text { plant }\end{array}$ & $\begin{array}{c}\text { Number of } \\
\text { clusters per } \\
\text { plant }\end{array}$ & $\begin{array}{c}\text { Number of } \\
\text { fruits per } \\
\text { cluster }\end{array}$ & $\begin{array}{c}\text { Number of } \\
\text { fruits per } \\
\text { plant }\end{array}$ & $\begin{array}{l}\text { Average fruit } \\
\text { weight (g) }\end{array}$ \\
\hline Replications & \begin{tabular}{|l|}
1 \\
\end{tabular} & 0.28 & 87.97 & 2.94 & $35.97 *$ & 0.0004 & 25.81 & 4.82 \\
\hline Crosses & 44 & $15.74 *$ & $1030.14 * *$ & $10.53 * *$ & $131.38 * *$ & $0.37 * *$ & $800.03 * *$ & $623.41 * *$ \\
\hline Line Effect & 14 & $31.64 * *$ & 1522.72 & 13.26 & 158.40 & 0.31 & 1231.53 & $1167.18 * *$ \\
\hline Tester Effect & 2 & 1.81 & 339.39 & $34.47 *$ & 166.10 & 0.05 & 35.04 & 737.02 \\
\hline Line $*$ Tester Effect & 28 & 8.79 & $833.18 * *$ & $7.46 * *$ & $115.39 * *$ & 0.42 & $638.91 * *$ & $343.41 * *$ \\
\hline Error & 44 & 8.60 & 27.64 & 0.98 & 8.12 & $0.04 * *$ & 17.87 & 12.26 \\
\hline Total & 89 & 12.04 & 523.93 & 5.73 & 69.37 & 0.20 & 404.65 & 314.32 \\
\hline
\end{tabular}




\begin{tabular}{|c|c|c|c|c|c|c|c|c|}
\hline $\begin{array}{c}\text { Source of } \\
\text { Variations }\end{array}$ & $\begin{array}{c}\text { Degrees of } \\
\text { freedom }\end{array}$ & $\begin{array}{c}\text { Polar length of } \\
\text { fruit }(\mathbf{m m})\end{array}$ & $\begin{array}{c}\text { Equatorial length of } \\
\text { fruit }(\mathbf{m m})\end{array}$ & $\begin{array}{c}\text { Fruit shape } \\
\text { index }\end{array}$ & $\begin{array}{c}\text { Pericarp } \\
\text { thickness (mm) }\end{array}$ & $\begin{array}{c}\text { No. of } \\
\text { locules }\end{array}$ & $\begin{array}{c}\text { TSS (\% } \\
\text { brix) }\end{array}$ & $\begin{array}{c}\text { Fruit yield per } \\
\text { plant }(\mathbf{k g})\end{array}$ \\
\hline Replications & 1 & 0.10 & 17.64 & 0.004 & 0.07 & 0.001 & 0.24 & 0.01 \\
\hline Crosses & 44 & $57.57^{* *}$ & $147.20^{* *}$ & $0.023^{* *}$ & $2.53^{* *}$ & $1.72^{* *}$ & $1.22^{* *}$ & $0.83^{* *}$ \\
\hline Line Effect & 14 & $101.39^{* *}$ & $266.96^{*}$ & 0.021 & 2.94 & 1.62 & 1.42 & $1.80^{* *}$ \\
\hline Tester Effect & 2 & 50.70 & 2.45 & 0.036 & 4.82 & 1.14 & 0.70 & $1.44^{*}$ \\
\hline Line * Tester Effect & 28 & $36.15^{* *}$ & $97.65^{* *}$ & $0.023^{* *}$ & $2.17 * *$ & $1.82^{* *}$ & $1.16^{* *}$ & $0.30^{* *}$ \\
\hline Error & 44 & 5.98 & 5.06 & 0.005 & 0.10 & 0.17 & 0.06 & 0.04 \\
\hline Total & 89 & 31.42 & 75.47 & 0.014 & 1.30 & 0.93 & 0.64 & 0.43 \\
\hline
\end{tabular}

* - Significant at $5 \%$ level ** - Significant at $5 \%$ level

Table 3: Estimates of variance components as reference to the prevailing gene action for yield and yield attributing characters in tomato

\begin{tabular}{|c|c|c|c|c|}
\hline Sl. No. & Traits & $\sigma^{2}$ gca & $\sigma^{2}$ sca & $\sigma^{2}$ gca $/ \sigma^{2}$ sca \\
\hline 1 & Days to $50 \%$ flowering & $0.43 * *$ & -0.06 & -7.17 \\
\hline 2 & Plant height $(\mathrm{cm})$ & $50.55^{*}$ & $406.07 * *$ & 0.12 \\
\hline 3 & No. of primary branches per plant & $0.09 * *$ & $0.21 * *$ & 0.43 \\
\hline 4 & No. of secondary branches per plant & $1.28 * *$ & $3.37 * *$ & 0.38 \\
\hline 5 & No. of clusters per plant & $8.61 *$ & $54.07 * *$ & 0.16 \\
\hline 6 & No. of fruits per cluster & 0.008 & $0.19 * *$ & 0.04 \\
\hline 7 & No. of fruits per plant & 34.29 & $311.41 * *$ & 0.11 \\
\hline 8 & Average fruit weight $(\mathrm{g})$ & $52.34 * *$ & $166.71 * *$ & 0.31 \\
\hline 9 & Polar length of fruit (mm) & $3.79 * *$ & $14.17 * *$ & 0.27 \\
\hline 10 & Equatorial length of fruit (mm) & $7.13^{*}$ & $45.63 * *$ & 0.16 \\
\hline 11 & Fruit shape index & 0.001 & $0.006^{*}$ & 0.17 \\
\hline 12 & Pericarp thickness $(\mathrm{mm})$ & 0.06 & $0.80 * *$ & 0.08 \\
\hline 13 & No. of locules per fruit & $0.21 *$ & $1.02 * *$ & 0.21 \\
\hline 14 & TSS (\% brix $)$ & 0.055 & $0.54 * *$ & 0.10 \\
\hline 15 & Fruit yield per plant (kg) & $0.088 * *$ & $0.13 * *$ & 0.68 \\
\hline
\end{tabular}

* - Significant at $5 \%$ level ** - Significant at $1 \%$ level

Table 4: Estimates of general combining ability effects of parents for yield and its attributing traits in tomato

\begin{tabular}{|c|c|c|c|c|c|c|c|c|c|c|c|c|c|c|}
\hline Parents & $\begin{array}{c}\text { Days to } \\
50 \% \\
\text { flowering }\end{array}$ & $\begin{array}{c}\text { Plant } \\
\text { height } \\
\text { (cm) }\end{array}$ & $\begin{array}{c}\text { No. of } \\
\text { branches } \\
\text { per plant }\end{array}$ & $\begin{array}{l}\text { No. of } \\
\text { clusters }\end{array}$ & \begin{tabular}{|c|} 
No. of \\
fruits \\
per \\
cluster
\end{tabular} & $\begin{array}{l}\text { Total } \\
\text { no. of } \\
\text { fruits }\end{array}$ & \begin{tabular}{|c} 
Average \\
fruit \\
weight \\
(g)
\end{tabular} & \begin{tabular}{|c|}
$\begin{array}{c}\text { Polar } \\
\text { length of } \\
\text { fruit } \\
\text { (mm) }\end{array}$ \\
\end{tabular} & $\begin{array}{c}\text { Equatorial } \\
\text { length of } \\
\text { fruit (mm) }\end{array}$ & $\begin{array}{c}\text { Fruit } \\
\text { shape } \\
\text { index }\end{array}$ & $\begin{array}{c}\text { Pericarp } \\
\text { thickness } \\
(\mathrm{mm})\end{array}$ & $\begin{array}{l}\text { No. of } \\
\text { locules }\end{array}$ & $\begin{array}{c}\text { TSS (\% } \\
\text { brix })\end{array}$ & $\begin{array}{c}\text { Yield } \\
\text { per } \\
\text { plant } \\
(\mathrm{kg})\end{array}$ \\
\hline \multicolumn{15}{|c|}{ Lines } \\
\hline L1 & -1.09 & 0.963 & -0.53 & -1.39 & $0.44 * *$ & $18.48 * *$ & -2.08 & 1.11 & 0.03 & 0.01 & -0.02 & 0.27 & $0.46^{* * *}$ & $0.58 * *$ \\
\hline $\mathrm{L} 2$ & -0.42 & $-10.25 * *$ & -0.03 & 0.59 & -0.13 & $-3.78^{*}$ & $-5.25 * *$ & -2.08 & $-4.67 * *$ & 0.05 & 0.12 & -0.22 & 0.19 & $-0.79 * *$ \\
\hline L3 & $6.08 * *$ & $-26.83 * *$ & $2.31 * *$ & $-5.38 * *$ & -0.13 & $24.19 * *$ & $-17.99 * *$ & $-2.99 *$ & $-4.82 * *$ & 0.06 & 0.15 & $1.34 * *$ & $1.14 * *$ & 0.0001 \\
\hline L4 & -0.76 & $-19.60 * *$ & $-2.19 * *$ & -1.66 & 0.09 & -1.88 & $12.07 * *$ & $4.84 * *$ & $4.42 * *$ & 0.01 & 0.13 & -0.37 & $-0.77 * *$ & $-0.26 * *$ \\
\hline L5 & 1.08 & $4.45^{*}$ & $-1.04 * *$ & 1.95 & -0.07 & $9.47 * *$ & $8.54 * *$ & 1.97 & $2.27 *$ & -0.01 & $0.60 * *$ & 0.21 & -0.15 & $0.48 * *$ \\
\hline L6 & -0.089 & $10.84 * *$ & 0.59 & 0.89 & $-0.24 * *$ & $-4.83 * *$ & $15.05 * *$ & $4.40 * *$ & $7.16 * *$ & -0.05 & $0.57 * *$ & -0.04 & $-0.42 * *$ & $0.212 * *$ \\
\hline L7 & -1.09 & $-6.95 * *$ & 0.11 & $-2.29 *$ & \begin{tabular}{|l|}
0.14 \\
\end{tabular} & 1.05 & $8.41 * *$ & 1.36 & $2.32 *$ & -0.02 & $0.38 *$ & $-0.51 *$ & -0.04 & $0.70 * *$ \\
\hline L8 & 1.41 & $-12.55 * *$ & $-0.76^{*}$ & $-4.85 * *$ & $0.37 * *$ & $\begin{array}{c}- \\
21.53^{* * *}\end{array}$ & $-12.86^{* *}$ & $-3.11^{* *}$ & $-5.05^{* *}$ & 0.01 & $-0.75 * *$ & $\begin{array}{c}- \\
0.54 * * \\
\end{array}$ & $-0.32 * *$ & $-0.52 * *$ \\
\hline L9 & -2.25 & $21.74 * *$ & $1.30 * *$ & $6.86 * *$ & $-0.19 *$ & $21.40 * *$ & $18.93 * *$ & 1.40 & $5.65 * *$ & -0.08 & $0.33^{*}$ & $0.52 * *$ & $0.27 *$ & $0.51 * *$ \\
\hline L10 & $-3.75 * *$ & $9.18 * *$ & $2.64 * *$ & $3.73 * *$ & $|-0.33 * *|$ & $11.92 * *$ & $4.97 * *$ & 0.87 & -0.10 & 0.03 & -0.23 & -0.09 & -0.12 & $0.29 * *$ \\
\hline L11 & -2.09 & $16.25^{* * *}$ & -0.17 & $-4.12 * *$ & $-0.27 * *$ & -2.55 & 0.38 & -1.11 & $4.04 * *$ & -0.08 & $-0.83 * *$ & $0.56 * *$ & $-0.46 * *$ & 0.128 \\
\hline L12 & -0.42 & $22.41^{* * *}$ & -0.03 & $3.62 * *$ & |0.17* & $5.87 * *$ & 1.58 & 1.95 & $4.12 * *$ & -0.04 & $0.78 * *$ & \begin{tabular}{|l|}
-0.21 \\
\end{tabular} & -0.20 & 0.13 \\
\hline L13 & 2.08 & $-18.08 * *$ & $-2.28 * *$ & $-7.98 * *$ & -0.002 & $\begin{array}{c}-23.29 \\
* *\end{array}$ & $5.76^{* *}$ & 1.03 & $3.49 * *$ & -0.05 & $0.66 * *$ & -0.13 & 0.05 & $-0.95 * *$ \\
\hline L14 & 1.58 & $16.73^{* * *}$ & $1.65 * *$ & $11.98^{* *}$ & 0.15 & $12.88 * *$ & $-35.54 * *$ & $-12.19 * *$ & $-19.81 * *$ & $0.14 * *$ & $-1.87 * *$ & $\begin{array}{c}- \\
0.66 * * \\
\end{array}$ & $0.65 * *$ & $-0.85 * *$ \\
\hline L15 & -0.25 & $-8.30^{* *}$ & $-1.36^{* *}$ & -1.94 & -0.03 & 0.98 & -1.98 & $2.53^{*}$ & 0.93 & 0.02 & -0.04 & \begin{tabular}{|l|}
-0.14 \\
\end{tabular} & $-0.27 *$ & $0.33^{* * *}$ \\
\hline SEm+ & 1.22 & 1.87 & 0.34 & 1.09 & 0.10 & 1.64 & 1.29 & 1.14 & 1.03 & 0.04 & 0.14 & 0.19 & 0.11 & 0.07 \\
\hline $\begin{array}{c}\mathrm{CD} \text { at } 5 \% \\
\text { female }\end{array}$ & 2.46 & 3.77 & 0.69 & 2.21 & 0.16 & 3.30 & 2.60 & 2.29 & 2.08 & 0.09 & 0.29 & 0.38 & 0.23 & 0.15 \\
\hline $\begin{array}{c}\mathrm{CD} \text { at } 1 \% \\
\text { female }\end{array}$ & 3.28 & 5.04 & 0.93 & 2.96 & 0.22 & 4.41 & 3.48 & 3.07 & 2.78 & 0.12 & 0.39 & 0.51 & 0.30 & 0.19 \\
\hline \multicolumn{15}{|c|}{ Testers } \\
\hline CLN2768A & -0.09 & -0.95 & $0.88 * *$ & $-1.09 *$ & 0.02 & 0.78 & $-4.01 * *$ & $-1.49 * *$ & 0.18 & $-0.04 *$ & $-0.44 * *$ & 0.05 & -0.05 & -0.03 \\
\hline CLN2777H & 0.28 & $3.73 * *$ & $0.31 *$ & $2.70 * *$ & 0.03 & -1.23 & $-1.54 *$ & 0.67 & -0.33 & 0.02 & 0.10 & 0.16 & 0.17 ** & $-0.20 * *$ \\
\hline AVTO1219 & -0.19 & $-2.78 * *$ & $-1.19^{* * *}$ & $-1.60^{* *}$ & -0.05 & 0.46 & $5.54 * *$ & 0.83 & 0.15 & 0.02 & $0.33 * *$ & $-0.22 *$ & $-0.12 *$ & $0.23 * *$ \\
\hline SEm+ & 1.19 & 0.84 & 0.15 & 0.49 & 0.03 & 0.73 & 0.58 & 0.51 & 0.46 & 0.02 & 0.06 & 0.08 & 0.05 & 0.03 \\
\hline $\mathrm{CD}$ at $5 \%$ male & 2.41 & 1.69 & 0.31 & 0.99 & 0.07 & 1.47 & 1.16 & 1.02 & 0.93 & 0.04 & 0.13 & 0.17 & 0.10 & 0.06 \\
\hline $\mathrm{CD}$ at $1 \%$ male & 3.22 & 2.25 & 0.42 & 1.32 & 0.09 & 1.97 & 1.55 & 1.37 & 1.24 & 0.05 & 0.17 & 0.23 & 0.14 & 0.09 \\
\hline
\end{tabular}

* - Significant at $5 \%$ level ** - Significant at $5 \%$ level 
Table 5: Estimates of specific combining ability effects of $F_{1}$ hybrids for yield and its attributing traits in tomato

\begin{tabular}{|c|c|c|c|c|c|c|c|c|c|c|c|c|c|c|c|}
\hline $\begin{array}{l}\text { Experimental } \\
\text { hybrids }\end{array}$ & $\begin{array}{c}\text { Days to } \\
\mathbf{5 0 \%} \\
\text { flowering }\end{array}$ & $\begin{array}{c}\text { Plant } \\
\text { height } \\
(\mathbf{c m})\end{array}$ & $\begin{array}{c}\text { No. of } \\
\text { primary } \\
\text { branches }\end{array}$ & $\begin{array}{c}\text { No. of } \\
\text { secondary } \\
\text { branches }\end{array}$ & $\left|\begin{array}{c}\text { No. of } \\
\text { clusters }\end{array}\right|$ & $\begin{array}{l}\text { No. of } \\
\text { fruits } \\
\text { per } \\
\text { cluster }\end{array}$ & $\begin{array}{l}\text { Total } \\
\text { no. of } \\
\text { fruits }\end{array}$ & $\begin{array}{l}\text { Average } \\
\text { fruit } \\
\text { weight } \\
\text { (g) }\end{array}$ & \begin{tabular}{|c|}
$\begin{array}{c}\text { Polar } \\
\text { length } \\
\text { of fruit } \\
(\mathrm{mm})\end{array}$ \\
\end{tabular} & $\begin{array}{l}\text { Equatorial } \\
\text { length of } \\
\text { fruit }(\mathbf{m m})\end{array}$ & $\begin{array}{l}\text { Fruit } \\
\text { shape } \\
\text { index }\end{array}$ & $\begin{array}{c}\text { Pericarp } \\
\text { thicknes } \\
(\mathbf{m m})\end{array}$ & $\begin{array}{l}\text { No. of } \\
\text { locules }\end{array}$ & $\begin{array}{c}\text { TSS } \\
(\% \\
\text { brix })\end{array}$ & $\begin{array}{c}\text { Yield } \\
\text { per } \\
\text { plant } \\
(\mathrm{kg})\end{array}$ \\
\hline L1 x T1 & -1.58 & $7.35 *$ & 0.05 & $-1.21 *$ & .18 & -0.02 & 1.06 & $-5.17 *$ & 3.22 & -1.31 & 0.092 & -0.11 & -0.15 & $0.83 * *$ & -0.03 \\
\hline & & & & & & -0.03 & & $-14.16^{* * *}$ & -8.19 ** & & & & -0.48 & 0.29 & -0.10 \\
\hline L1 x T3 & 0.52 & $-8.32 *$ & $-0.38 * *$ & 0.86 & -0.39 & 0.05 & -2.63 & $19.33^{* *}$ & $4.97 *$ & $8.87 * *$ & -0.06 & $0.90 * *$ & 0.63 & $1.11 * *$ & 0.132 \\
\hline L2 x T1 & 0.76 & $26.22 * *$ & -0.10 & $2.78 * *$ & 3.33 & $-0.65^{* *}$ & 0.52 & $22.00 * *$ & $7.93 * *$ & $9.63 * *$ & -0.03 & $1.33 * *$ & 0.46 & $\overline{9} \cdot * *$ & $0.26^{*}$ \\
\hline L2 x T2 & 061 & .73 & 222 & $15 * *$ & $-5.78 * *$ & 0.04 & $-6.27 *$ & $5.89 *$ & 1.14 & $663 * *$ & 0.12 & $0.84 * *$ & $0.80^{*}$ & 0.20 & 0.25 \\
\hline L2 $x$ T3 & -0.14 & $\begin{array}{c}-27.95 \\
* *\end{array}$ & $0.32 *$ & 0.36 & 2.45 & $0.61 * *$ & $5.74 *$ & $-27.89 * *$ & $-9.08 * *$ & $-16.26 * *$ & 0.15 & $-2.17 * *$ & $-1.27 * *$ & $0.78 * *$ & $-0.51 * *$ \\
\hline L3 x T1 & & -5.55 & $48 * *$ & & -3.05 & -0.15 & $-11.86 * *$ & $5.54 *$ & 0.43 & & -0.14 & & $1.29 * *$ & $0.75 * *$ & $-0.61 * *$ \\
\hline $\mathrm{L} 3 \times \mathrm{T} 2$ & -1 & $09 *$ & $-0.35 *$ & & $-5.99 * *$ & $-0.46^{* *}$ & $-16.49 * *$ & $-7.23 * *$ & -0.96 & $7.59 * *$ & $-0.19 *$ & $-0.68 * *$ & $1.04 * *$ & 0.19 & $-0.27 *$ \\
\hline L3 $\times$ T3 & 0.36 & $12.63 * *$ & $0.83 * *$ & $-1.72 * *$ & $9.04 * *$ & $0.61 * *$ & $28.36 * *$ & 1.69 & 0.53 & $-13.69 * *$ & $0.33 * *$ & $0.753 * *$ & $-2.33 * *$ & $|-\overline{-}|$ & $0.88 * *$ \\
\hline L4 x T1 & & $98 * *$ & & & -0.42 & $-0.47 * *$ & $-6.08 *$ & -3.37 & $-5.29 *$ & $-3.704 *$ & 0.03 & & 0.61 & 0.05 & $-0.26^{*}$ \\
\hline $\mathrm{L} 4 \mathrm{x}$ T2 & -2.28 & $10.78 * *$ & -0.27 & -0.98 & $-4.87 *$ & $0.32 *$ & 0.34 & -3.16 & 0.57 & $-4.41 *$ & 0.09 & $0.86^{* *}$ & -0.496 & $\begin{array}{l}-0.62 \\
* *\end{array}$ & -0.03 \\
\hline L4 x T3 & & & & & $29 * *$ & 0.17 & $741 *$ & $6.52 * *$ & $4.72 *$ & & -0.06 & & -0.117 & $0.58 * *$ & $0.29 *$ \\
\hline L5 x T1 & 1.256 & $\begin{array}{c}-33.18 \\
* * \\
\end{array}$ & $-0.50 * *$ & -0.69 & $-9.58 * *$ & $0.49 * *$ & $-21.38 * *$ & $-6.71 * *$ & -1.38 & $-4.07 *$ & 0.049 & $0.53^{*}$ & $\begin{array}{c}-1.22 \\
* * \\
\end{array}$ & $\begin{array}{c}- \\
0.58 * *\end{array}$ & $-0.33 *$ \\
\hline & & & & & & 0.15 & $73 * *$ & 11.09 ** & 250 & & קר? & & & \begin{tabular}{|l|}
-0.35 \\
\end{tabular} & 0.19 \\
\hline & & $35 * *$ & & & $7.63 * *$ & $-0.64 * *$ & $12.64 * *$ & -4.38 & -1.21 & & .07 & & $1.79 * *$ & $0.93 * *$ & 0.14 \\
\hline & & $13 * *$ & & & & -0.04 & 0.07 & $-8.08 * *$ & -0.44 & & .08 & & $-0.72 *$ & $-0.50 *$ & -0.07 \\
\hline & & & & & & .14 & & -3.56 & -2.60 & & 02 & & 17 & 0.08 & 0.04 \\
\hline & & & & & & & & & 3. & & & & & $0.43^{*}$ & 0.03 \\
\hline & & & & & & & & & - & & & & & 0.34 & -0.09 \\
\hline & & & & & $0.42-1$ & -0.13 & -0.75 & $-12.32 * *$ & -1.44 & & .02 & & -0.36 & 0.09 & 0.08 \\
\hline L7x T3 & 0.02 & & 15 & -0.31 & -2.78 & 0.15 & $6.76^{*}$ & $7.42 * *$ & 1.64 & 0.46 & 0.01 & $-0.76 * *$ & 0.35 & $-0.43 *$ & 0.01 \\
\hline & & $2.52 * *$ & & & & .05 & $22 * *$ & $.24 * *$ & -3.30 & 170: & .02 & & 28 & $0.51 *$ & $0.79^{* *}$ \\
\hline & & $-24.17 * *$ & & & $-5.57 * *$ & -0.16 & -16.89 *** & $6.63 * *$ & $5.36 * *$ & & 10 & & -0.33 & -0.23 & $-0.34 *$ \\
\hline L8 x T3 & & & & & & 0.11 & & 0.61 & -2.06 & 3.37 & -0.11 & -0.25 & 0.05 & -0.28 & $\begin{array}{c}-0.46 \\
* *\end{array}$ \\
\hline L9 x T1 & & & & $-1.79 * *$ & $-9.94 * *$ & 0.21 & $-29.11 * *$ & 1.40 & 0.50 & 0.01 & 0.03 & $0.75 * *$ & -0.03 & 0.16 & -0.01 \\
\hline L9 x T2 & -0.28 & $-6.97 *$ & -0.21 & 0.77 & 1.37 & $-0.59 * *$ & $12.40 * *$ & -0.30 & -3.52 & $-4.03 *$ & 0.01 & -0.17 & -0.14 & $\begin{array}{c}-0.78 \\
* *\end{array}$ & 0.05 \\
\hline & & & & & $57 * *$ & $0.39 * *$ & $16.71 * *$ & & 3.02 & & 0.03 & & 0.17 & $0.62 * *$ & -0.04 \\
\hline & & & & & & & & $-19.58 * *$ & $-4.20^{*}$ & & & & $67 *$ & $1.29 * *$ & 0.03 \\
\hline & & & & & -0 . & $0.74 * *$ & 3.5 & -1.73 & 2.36 & & 0.13 & & 0.32 & -0.29 & -0.09 \\
\hline & -1.31 & $13.12 * *$ & 0.26 & -0.31 & -1.58 & $-0.29 *$ & 5.34 & $21.31 * *$ & 1.84 & $7.75^{* *}$ & -0.08 & $1.08^{* *}$ & 0.35 & $1.01 * *$ & 0.06 \\
\hline $\mathrm{L} 11 \times \mathrm{T} 1$ & -0.08 & -0.37 & 0.27 & $0.5 J$ & -1.26 & $0.45^{* *}$ & $7.34 *$ & 0.22 & -0.66 & 0.66 & 0.00 & $-0.69 * *$ & $-0.82 *$ & $-0.47 *$ & 0.17 \\
\hline
\end{tabular}

Table 4: Contd....

\begin{tabular}{|c|c|c|c|c|c|c|c|c|c|c|c|c|c|c|c|}
\hline $\begin{array}{c}\text { Experimental } \\
\text { hybrids }\end{array}$ & $\begin{array}{c}\text { Days to } \\
50 \% \\
\text { flowering }\end{array}$ & $\begin{array}{c}\text { Plant } \\
\text { height } \\
(\mathrm{cm})\end{array}$ & $\begin{array}{c}\text { No. of } \\
\text { primary } \\
\text { branches }\end{array}$ & $\begin{array}{c}\text { No. of } \\
\text { secondary } \\
\text { branches }\end{array}$ & $\begin{array}{l}\text { No. of } \\
\text { clusters }\end{array}$ & \begin{tabular}{|c|}
$\begin{array}{c}\text { No. of } \\
\text { fruits } \\
\text { per } \\
\text { cluster }\end{array}$ \\
\end{tabular} & $\begin{array}{l}\text { Total } \\
\text { no. of } \\
\text { fruits }\end{array}$ & \begin{tabular}{|c|} 
Average \\
fruit \\
weight \\
(g) \\
\end{tabular} & \begin{tabular}{|c|}
$\begin{array}{c}\text { Polar } \\
\text { length } \\
\text { of fruit } \\
(\mathrm{mm})\end{array}$ \\
\end{tabular} & $\begin{array}{l}\text { Equatorial } \\
\text { length of } \\
\text { fruit }(\mathrm{mm})\end{array}$ & $\begin{array}{l}\text { Fruit } \\
\text { shape } \\
\text { index }\end{array}$ & $\begin{array}{c}\text { Pericarp } \\
\text { thickness } \\
(\mathrm{mm})\end{array}$ & $\begin{array}{l}\text { No. of } \\
\text { locules }\end{array}$ & $\begin{array}{c}\text { TSS } \\
(\% \\
\text { brix })\end{array}$ & $\begin{array}{l}\text { Yield } \\
\text { per } \\
\text { plant } \\
(\mathrm{kg})\end{array}$ \\
\hline $\mathrm{L} 11 \times \mathrm{T} 2$ & -0.44 & $21.52 * *$ & 0.101 & -0.50 & $6.29 * *$ & $-0.41 * *$ & $12.35 * *$ & \begin{tabular}{|l|}
4.48 \\
\end{tabular} & \begin{tabular}{|l|}
1.97 \\
\end{tabular} & 2.51 & -0.01 & $0.65^{*}$ & $0.87 *$ & $0.46^{*}$ & 0.12 \\
\hline L11 x T3 & 0.52 & $\begin{array}{c}-21.16 \\
* *\end{array}$ & $-0.368 *$ & 0.15 & $-5.04 *$ & -0.04 & $-19.69 * *$ & $-4.71 *$ & -1.31 & -3.17 & 0.00 & 0.04 & -0.05 & 0.01 & $-0.29 *$ \\
\hline L12 x T1 & -2.74 & $\begin{array}{c}-15.25 \\
* *\end{array}$ & -0.09 & $-1.21 *$ & -2.46 & $-0.29 *$ & -1.68 & $16.14 * *$ & 3.03 & $4.86^{* *}$ & -0.01 & $1.29 * *$ & -0.05 & -0.38 & -0.21 \\
\hline L12 x T2 & 1.89 & -3.13 & 0.18 & 0.85 & -0.35 & 0.24 & -2.07 & 0.75 & 1.95 & 2.31 & 0.00 & -0.48 & 0.34 & $0.80 * *$ & 0.02 \\
\hline L12 x T3 & 0.86 & $18.38^{* *}$ & -0.09 & 0.36 & 2.81 & 0.06 & 3.74 & $-16.88^{* *}$ & $-4.98 *$ & $-7.18^{* *}$ & 0.01 & $-0.82 * *$ & -0.29 & $-0.43 *$ & 0.19 \\
\hline $\mathrm{L} 13 \times \mathrm{T} 1$ & 2.26 & $39.62 * *$ & $0.60 * *$ & $2.27 * *$ & $4.67 *$ & 0.18 & $11.09 * *$ & $-8.26^{* *}$ & \begin{tabular}{|l|}
0.06 \\
\end{tabular} & $-4.22^{*}$ & 0.08 & $-0.77 * *$ & -0.63 & 0.22 & 0.04 \\
\hline $\mathrm{L} 13 \times \mathrm{T} 2$ & 2.89 & $-23.85 * *$ & $-0.57 * *$ & $-1.89 * *$ & $-4.09 *$ & 0.04 & $-8.49 * *$ & 2.57 & -2.79 & 1.64 & -0.07 & -0.02 & 0.29 & $\begin{array}{c}- \\
0.79 * *\end{array}$ & $-0.45^{* *}$ \\
\hline L13 x T3 & $-5.14 *$ & $\begin{array}{c}-15.76 \\
* *\end{array}$ & -0.04 & -0.38 & -0.58 & -0.21 & -2.59 & $5.69 *$ & 2.74 & 2.58 & -0.01 & $0.79 * *$ & 0.34 & $0.58 * *$ & $0.41 * *$ \\
\hline L14 x T1 & 0.76 & $-21.91 * *$ & $0.53 * *$ & 0.55 & $14.14 * *$ & 0.27 & $25.76^{* *}$ & $5.17 *$ & 1.65 & 2.01 & -0.03 & -0.06 & 0.05 & $\begin{array}{c}- \\
1.03 * *\end{array}$ & 0.23 \\
\hline $\mathrm{L} 14 \times \mathrm{T} 2$ & 2.39 & $12.55 * *$ & 0.26 & $1.38^{*}$ & $4.81 *$ & -0.03 & $21.42 * *$ & -2.26 & 0.25 & -1.57 & 0.08 & 0.14 & -0.21 & $0.55 * *$ & $0.36^{* * *}$ \\
\hline L14 x T3 & -3.14 & $9.37 * *$ & $-0.79 * *$ & $-1.93 * *$ & $\begin{array}{c}- \\
18.95 * *\end{array}$ & -0.24 & $-47.18 * *$ & -2.91 & -1.90 & -0.44 & -0.05 & -0.07 & 0.17 & $0.48 *$ & $-0.59 * *$ \\
\hline L15 x T1 & -0.91 & $10.12 * *$ & 0.23 & 0.12 & -1.26 & $0.45 * *$ & 3.81 & 2.97 & -1.34 & 0.78 & -0.03 & $-1.38 * *$ & $1.58 * *$ & -0.20 & 0.10 \\
\hline $\mathrm{L} 15 \times \mathrm{T} 2$ & -1.28 & $-7.00^{*}$ & 0.12 & 1.19 & 3.12 & $0.44^{* *}$ & 2.32 & $13.32 * *$ & 3.30 & 3.34 & 0.01 & $1.82^{* * *}$ & $-1.23 * *$ & 0.38 & 0.16 \\
\hline L15 x T3 & 2.19 & -3.05 & $-0.35 *$ & $-1.31 *$ & -1.86 & $-0.89 * *$ & $-6.17 *$ & $-16.29 * *$ & -1.97 & $-4.13 *$ & 0.03 & -0.44 & \begin{tabular}{|l|}
-0.35 \\
\end{tabular} & -0.18 & $-0.26^{*}$ \\
\hline SEm+ & 2.11 & 3.24 & 0.14 & 0.59 & 1.90 & \begin{tabular}{|l|}
0.14 \\
\end{tabular} & 2.83 & 2.23 & 1.97 & 1.78 & 0.07 & 0.25 & 0.33 & 0.19 & 0.12 \\
\hline $\mathrm{CD}$ at $5 \%$ & 4.26 & 6.54 & 0.29 & 1.21 & 3.84 & 0.28 & 5.72 & 4.51 & 3.99 & 3.60 & 0.15 & 0.50 & 0.67 & 0.39 & 0.25 \\
\hline $\mathrm{CD}$ at $1 \%$ & 5.68 & 8.73 & 0.38 & 1.61 & 5.12 & 0.30 & 7.63 & 6.02 & 5.31 & 4.81 & 0.20 & 0.67 & 0.89 & 0.52 & 0.34 \\
\hline
\end{tabular}

* - Significant at 5\% level ** - Significant at 5\% level 
Table 6: Promising crosses of tomato on the basis of mid parent heterosis.

\begin{tabular}{|c|c|}
\hline Characters & Best crosses for MP heterosis (percent) \\
\hline Days to $50 \%$ flowering & L3 x T1 \\
\hline Plant height $(\mathrm{cm})$ & L4 x T3 (35.26) \\
\hline Number of branches per plant & $\mathrm{L} 10 \times \mathrm{T} 2(60.53)$ \\
\hline Number of clusters per plant & L10 x T2 (80.01) \\
\hline Number of fruits per cluster & L5 x T1 (40.56) L14 x T1 (40.56) \\
\hline Total number of fruits per plant & $\mathrm{L} 2 \times \mathrm{T} 1(77.28)$ \\
\hline Average fruit weight $(\mathrm{g})$ & L1 x T3 (49.66) \\
\hline Polar length of fruit (mm) & L1 x T3 (17.85) \\
\hline Equatorial length of fruit (mm) & L1 x T3 (37.28) \\
\hline Fruit shape index & L3 x T3 (35.82) \\
\hline Pericarp thickness $(\mathrm{mm})$ & L13 x T3 (48.34) \\
\hline Number of locules & L1 x T3 (50.43) \\
\hline TSS (\% brix) & L3 x T1 (23.24) \\
\hline Fruit yield per plant $(\mathrm{kg})$ & L4 x T3 (35.26) \\
\hline
\end{tabular}

Table 7: Mean of per cent disease incidence taken at 30, 60 and 90 DAT and disease reaction of the $\mathrm{F}_{1}$ hybrids developed by crossing 15 lines with 3 testers screened under natural condition for ToLCV disease during summer 2019

\begin{tabular}{|c|c|c|c|c|c|}
\hline Crosses & 30 DAT & $60 \mathrm{DAT}$ & 90 DAT & Mean of per cent disease incidence & Disease reaction \\
\hline L1 x T1 & 0.00 & 40.00 & 60.00 & 33.33 & $\mathrm{~T}$ \\
\hline L1 1 T2 & 20.00 & 60.00 & 80.00 & 53.33 & $\mathrm{~S}$ \\
\hline L1 x T3 & 40.00 & 60.00 & 80.00 & 60.00 & $\mathrm{~S}$ \\
\hline $\mathrm{L} 2 \times \mathrm{T} 1$ & 0.00 & 40.00 & 60.00 & 33.33 & $\mathrm{~T}$ \\
\hline $\mathrm{L} 2 \times \mathrm{T} 2$ & 0.00 & 40.00 & 60.00 & 33.33 & $\mathrm{~T}$ \\
\hline $\mathrm{L} 2 \times \mathrm{T} 3$ & 40.00 & 60.00 & 80.00 & 60.00 & $\mathrm{~S}$ \\
\hline L3 x T1 & 20.00 & 40.00 & 60.00 & 40.00 & $\mathrm{~T}$ \\
\hline L3 x T2 & 20.00 & 60.00 & 80.00 & 53.33 & $S$ \\
\hline L3 $x$ T3 & 0.00 & 40.00 & 60.00 & 33.33 & $\mathrm{~T}$ \\
\hline L4 x T1 & 20.00 & 40.00 & 40.00 & 33.33 & $\mathrm{~S}$ \\
\hline $\mathrm{L} 4 \times \mathrm{T} 2$ & 40.00 & 60.00 & 80.00 & 60.00 & $\mathrm{~S}$ \\
\hline $\mathrm{L} 4 \times \mathrm{T} 3$ & 0.00 & 40.00 & 60.00 & 33.33 & $\mathrm{~T}$ \\
\hline L5 x T1 & 0.00 & 60.00 & 80.00 & 46.67 & $\mathrm{~T}$ \\
\hline $\mathrm{L} 5 \times \mathrm{T} 2$ & 40.00 & 20.00 & 60.00 & 40.00 & $\mathrm{~T}$ \\
\hline $\mathrm{L} 5 \times \mathrm{T} 3$ & 20.00 & 60.00 & 80.00 & 53.33 & $\mathrm{~S}$ \\
\hline L6 x T1 & 20.00 & 60.00 & 60.00 & 46.67 & $\mathrm{~T}$ \\
\hline L6 x T2 & 0.00 & 20.00 & 40.00 & 20.00 & MR \\
\hline L6 x T3 & 0.00 & 40.00 & 60.00 & 33.33 & $\mathrm{~T}$ \\
\hline L7 x T1 & 40.00 & 60.00 & 100.00 & 66.67 & $\mathrm{~S}$ \\
\hline $\mathrm{L} 7 \times \mathrm{T} 2$ & 40.00 & 40.00 & 60.00 & 46.67 & $\mathrm{~T}$ \\
\hline $\mathrm{L} 7 \times \mathrm{T} 3$ & 0.00 & 40.00 & 60.00 & 33.33 & $\mathrm{~T}$ \\
\hline L8 x T1 & 0.00 & 20.00 & 40.00 & 20.00 & MR \\
\hline L8 x T2 & 40.00 & 60.00 & 60.00 & 53.33 & $\mathrm{~S}$ \\
\hline L8 $\times$ T3 & 20.00 & 40.00 & 60.00 & 40.00 & $\mathrm{~T}$ \\
\hline L9 x T1 & 40.00 & 60.00 & 60.00 & 53.33 & $\mathrm{~S}$ \\
\hline L9 $\times$ T2 & 40.00 & 60.00 & 80.00 & 60.00 & $\mathrm{~S}$ \\
\hline L9 x T3 & 20.00 & 40.00 & 60.00 & 40.00 & $\mathrm{~T}$ \\
\hline L10 x T1 & 0.00 & 40.00 & 60.00 & 33.33 & $\mathrm{~T}$ \\
\hline $\mathrm{L} 10 \times \mathrm{T} 2$ & 0.00 & 20.00 & 40.00 & 20.00 & MR \\
\hline L10 x T3 & 40.00 & 60.00 & 80.00 & 60.00 & $\mathrm{~S}$ \\
\hline $\mathrm{L} 11 \times \mathrm{T} 1$ & 0.00 & 40.00 & 60.00 & 33.33 & $\mathrm{~T}$ \\
\hline $\mathrm{L} 11 \times \mathrm{T} 2$ & 0.00 & 20.00 & 60.00 & 26.67 & $\mathrm{~T}$ \\
\hline L11 x T3 & 40.00 & 60.00 & 80.00 & 60.00 & $\mathrm{~S}$ \\
\hline L12 x T1 & 20.00 & 40.00 & 60.00 & 40.00 & $\mathrm{~T}$ \\
\hline $\mathrm{L} 12 \times \mathrm{T} 2$ & 20.00 & 40.00 & 60.00 & 40.00 & $\mathrm{~T}$ \\
\hline L12 x T3 & 0.00 & 20.00 & 40.00 & 20.00 & MR \\
\hline L13 x T1 & 0.00 & 40.00 & 60.00 & 33.33 & $\mathrm{~T}$ \\
\hline $\mathrm{L} 13 \times \mathrm{T} 2$ & 40.00 & 60.00 & 80.00 & 60.00 & $\mathrm{~S}$ \\
\hline L13 x T3 & 0.00 & 20.00 & 40.00 & 20.00 & MR \\
\hline L14 x T1 & 0.00 & 40.00 & 60.00 & 33.33 & $\mathrm{~T}$ \\
\hline L14 x T2 & 40.00 & 60.00 & 80.00 & 60.00 & $\mathrm{~S}$ \\
\hline L14 x T3 & 20.00 & 40.00 & 60.00 & 40.00 & $\mathrm{~T}$ \\
\hline L15 x T1 & 20.00 & 40.00 & 60.00 & 40.00 & $\mathrm{~T}$ \\
\hline $\mathrm{L} 15 \times \mathrm{T} 2$ & 0.00 & 40.00 & 60.00 & 33.33 & $\mathrm{~T}$ \\
\hline L15 x T3 & 0.00 & 40.00 & 60.00 & 33.33 & $\mathrm{~T}$ \\
\hline $1601-01$ & 40.00 & 60.00 & 80.00 & 60.00 & $\mathrm{~S}$ \\
\hline
\end{tabular}




\begin{tabular}{|c|c|c|c|c|c|}
\hline $1601-10$ & 40.00 & 80.00 & 80.00 & 66.67 & $\mathrm{~S}$ \\
\hline $1601-14$ & 20.00 & 60.00 & 80.00 & 53.33 & $S$ \\
\hline $1601-22$ & 40.00 & 60.00 & 100.00 & 66.67 & S \\
\hline $1601-24$ & 40.00 & 60.00 & 80.00 & 60.00 & $\mathrm{~S}$ \\
\hline $1601-36$ & 60.00 & 100.00 & 100.00 & 86.67 & $\mathrm{HS}$ \\
\hline 1601-37 & 40.00 & 60.00 & 80.00 & 60.00 & $\mathrm{~S}$ \\
\hline $1601-47$ & 40.00 & 60.00 & 100.00 & 66.67 & $\mathrm{~S}$ \\
\hline $1602-03$ & 60.00 & 80.00 & 100.00 & 80.00 & $\mathrm{HS}$ \\
\hline $1602-06$ & 0.00 & 60.00 & 100.00 & 53.33 & $\mathrm{~S}$ \\
\hline 1602-09 & 40.00 & 60.00 & 80.00 & 60.00 & $S$ \\
\hline $1602-11$ & 60.00 & 60.00 & 80.00 & 66.67 & $S$ \\
\hline $1602-15$ & 40.00 & 40.00 & 60.00 & 46.67 & $\mathrm{~T}$ \\
\hline $1602-21$ & 60.00 & 100.00 & 100.00 & 86.67 & $\mathrm{HS}$ \\
\hline $1602-50$ & 0.00 & 60.00 & 80.00 & 46.67 & $\mathrm{~T}$ \\
\hline CLN2768A & 0.00 & 20.00 & 20.00 & 0.00 & $\mathrm{R}$ \\
\hline CLN2777H & 0.00 & 0.00 & 0.00 & 0.00 & $\mathrm{R}$ \\
\hline AVTO-1219 & 0.00 & 0.00 & 0.00 & 0.00 & $\mathrm{R}$ \\
\hline
\end{tabular}

DAT: days after transplanting; R: resistant; MR: moderately resistance; T: tolerant; S: susceptible; HS: highly Susceptibl

Table 8: Crosses having high heterosis for yield per plant and its resistance reaction in tomato

\begin{tabular}{|c|c|c|}
\hline Crosses & Per see performance yield per plant & Resistance reaction \\
\hline L3 $\times$ T3 & 3.84 & $\mathrm{~T}$ \\
\hline $\mathrm{L} 1 \times \mathrm{T} 3$ & 3.69 & $\mathrm{MR}$ \\
\hline $\mathrm{L} 5 \times \mathrm{T} 3$ & 3.59 & $\mathrm{~T}$ \\
\hline $\mathrm{L} 10 \times \mathrm{T} 3$ & 3.32 & $\mathrm{~T}$ \\
\hline $\mathrm{L} 7 \times \mathrm{T} 2$ & 3.32 & $\mathrm{~T}$ \\
\hline $\mathrm{L} 7 \times \mathrm{T} 1$ & 3.31 & $\mathrm{~T}$ \\
\hline $\mathrm{L} 12 \times \mathrm{T} 3$ & 3.28 & $\mathrm{~T}$ \\
\hline $\mathrm{L} 1 \times \mathrm{T} 1$ & 3.26 & $\mathrm{~T}$ \\
\hline $\mathrm{L} 6 \times \mathrm{T} 3$ & 3.21 & $\mathrm{MR}$ \\
\hline $\mathrm{L} 9 \times \mathrm{T} 1$ & 3.20 & $\mathrm{~T}$ \\
\hline $\mathrm{L} 5 \times \mathrm{T} 2$ & 3.20 & $\mathrm{MR}$ \\
\hline $\mathrm{L} 15 \times \mathrm{T} 1$ & 3.13 & $\mathrm{MR}$ \\
\hline $\mathrm{L} 9 \times \mathrm{T} 2$ & 3.09 & $\mathrm{~T}$ \\
\hline $\mathrm{L} 15 \times \mathrm{T} 3$ & 3.04 & \\
\hline
\end{tabular}

\section{Conclusion}

It could be concluded from the present study that out of 15 lines seven lines viz., Seven lines L1, L5, L6, L7, L9, L10, L14 and L15 and the tester AVTO-1219 identified as a good combiners for most of the traits studied. Similarly four crosses viz., $\mathrm{L} 3 \times \mathrm{T} 3, \mathrm{~L} 8 \times \mathrm{T} 1, \mathrm{~L} 13 \times \mathrm{T} 3$ and $\mathrm{L} 14 \times \mathrm{T} 4$ are identified as the good specific combiner and highest per se performance for yield per plant. Similarly Crosses L3 $\times$ T3, L1 $\times$ T3 and L7 $\times$ T3 exhibited superior performance for yield compared to mid parent. Out of 45 hybrids evaluated for ToLCV disease resistance, five hybrids $\mathrm{L} 6 \times \mathrm{T} 2, \mathrm{~L} 7 \times \mathrm{T} 3, \mathrm{~L} 10 \times \mathrm{T} 2, \mathrm{~L} 12 \times$ T3 and L13 $\times$ T3 exhibited moderate resistance reaction. From this study, it may be concluded that the cross combinations $\mathrm{L} 3 \times \mathrm{T} 3, \mathrm{~L} 1 \times \mathrm{T} 3$ and $\mathrm{L} 5 \times \mathrm{T} 3$ could be included for further testing for exploitation of hybrid vigour in tomato in ToLCV affected areas.

\section{References}

1. Agarwal A, Arya DN, Ranjan R, Ahmed Z. Heterosis, combining ability and gene action for yield and quality traits in tomato (Solanum lycopersicum L.). Helix. 2014; 2:511- 515 .

2. Ahmad S, Quamruzzaman AKM, Uddin MN. Combining ability estimates of tomato (Solanum lycopersicum L.) in late summer. SAARC J Agri. 2009; 7(1):43-56.

3. Angadi A, Dharmatti PR. Heterosis for processing quality traits in tomato (Solanum lycopersicum L.). Res. J Agric. Sci. 2012; 3:1028-1030.
4. Asati BS, Singh G, Rai N, Chaturvedi AK. Heterosis and combining ability studies for yield and quality traits in tomato. Veg. Sci. 2007; 34:92-94.

5. Banerjee MK, Kalloo. Sources and inheritance of resistance to leaf curl virus in Lycopersicon. Theor. Appl. Genet. 1987a; 73:707-710.

6. Bhatt RP, Biswas VR, Kumar N. Heterosis, combining ability and genetics of vitamin $\mathrm{C}$, total soluble solids and yield in tomato (Lycopersicon esculentum Mill.) at 1700 m altitude. J Agri. Sci. 2000; 137(1):71-75.

7. Bhatt RP, Adhekari RS, Biswas VR, Kumar N. Genetical analysis for quantitative and qualitative traits in tomato (Lycopersicon esculentum Mill.) under open and protected environment. Indian J Genet. 2004; 64(2):125129.

8. Choudhary B, Punia RS, Sangha HS. Manifestation of hybrid vigour in $\mathrm{F}_{1}$ and its correlation in $\mathrm{F}_{2}$ generation of tomato (Lycopersicon esculentum Mill). Indian J Hort. 1965; 22:52-59.

9. Falconer DS. Introduction to quantitative genetics. $12^{\mathrm{a}}$ impression. Editorial CECSA, MEX, 1981.

10. Farzane A, Nemati H, Arouiee H, Kakhki AM, Vahdati $\mathrm{N}$. The estimate of combining ability and heterosis for yield and yield components in tomato (Lycopersicon esculentum Mill.). J Biol. Environ. Sci. 2012; 6(17):129134.

11. Hedrick UP, Booth NO. Mendelian characters in tomato. Proceedings of American Society. Hortic. Sci. 1968; 5:19-24. 
12. Himanshu G, Rai N, Singh RK, Singh DB. Expression of heterosis and combining abilities (Gca \& Sca) in inter specific crosses of tomato (Lycopersicon esculentum Mill.). J Plant Genet. Resour. 2008; 21(3):221-224.

13. Joshi A, Kohli UK. Combining ability and gene action studies for processing quality attributes in tomato (Lycopersicon esculentum Mill.). Indian J Hort. 2006; 3(3):289-293.

14. Kempthorne O. An introduction to genetic statistics, John Wiley and sons, Inc. New York, 1957.

15. Lapidot M, Friedmann M. Breeding for resistance to whitefly-transmitted geminiviruses. Ann. Appl. Biol. 2002; 140:109-127.

16. Makesh S, Puddan M, Bane MR, Ramaswamy N. Heterosis for some important quantitative traits in tomato. Res. Crops. 2003; 4(2):235-239.

17. Mali B, Patel AI. Combining ability study in tomato (Lycopersicon esculentum Mill.). Trends Biosci. 2014; 7(4):254-256.

18. Narasimhamurthy $\mathrm{YK}$, Gowda PHR. Line $\mathrm{x}$ tester analysis in tomato (Solanum lycopersicum L.) identification of superior parents for fruit quality and yield-attributing traits. Int. J Pl. Breed. 2013; 7(1):50-54.

19. Nitu K, Srivastava JP, Singh B, Singh B. Heterotic expression for yield and its component in tomato (Lycopersicon esculentum Mill.). Ann. Hort. 2010; 3(1):98-101.

20. Premlakshmi V, Thyagaraj T, Veeraragavathatham D, Arumugam T. Heterosis and combining ability analysis in tomato [Lycopersicon esculentum (Mill.) Wettsd] for yield and yield contributing traits. Vegetable Science. 2006; 33:5-9

21. Rick CM. Origin of cultivated tomato, current status and the problem. Proceedings of the $11^{\text {th }}$ International Botanical Congress, Seattle, 1969, 180.

22. Saleem MY, Asghar M, Iqbal Q, Rehman A, Akram M. Diallel analysis of yield and some yield components in tomato (Solanum lycopersicum L.). Pakistan J Bot. 2013; 45(4):1247-1250.

23. Shankar A, Reddy RVSK, Sujatha M, Pratap M. Combining ability and gene action studies for yield and yield contributing traits in tomato (Solanum lycopersicum L.). Helix. 2013; 6:431-435.

24. Sharif A, Bakhsh A, Arshad A, Haqqani AM, Najma S. Identification of genetically superior hybrids in chickpea (Cicer arietinum L.). Pak. J Bot. 2001; 33(4):403-409.

25. Singh AK, Asati BS. Combining ability and heterosis studies in tomato under bacterial wilt condition. Bangladesh J Agric. Res. 2011; 36(2):313-318.

26. Singh A, Gautam JPS, Upadyay M, Joshi A. Heterosis for yield and quality characters in tomato (Solanum lycopersicum L.). Crop Res. 2005; 29(2):285-287.

27. Singh B, Rai N, Singh RK, Singh MC, Singh AK, Chaturvedi AK. Heterosis, combining ability and gene action studies in tomato (Solanum lycopersicum L.). Veg. Sci. 2008; 35(2):132-135.

28. Singh B, Kaul S, Kumar D, Kumar V. Combining ability for yield and its contributing characters in tomato (Solanum lycopersicum L.). Indian J Hort. 2010; 67(1):50-55.

29. Tiwari A, Lal G. Studies on heterosis for quantitative and qualitative character in tomato (Lycopersicon esculentum Mill.). Prog. Hort. 2004; 36:122-127.

30. Yadav SK, Singh BK, Baranwal DK, Solankey SS. Genetic study of heterosis for yield and quality components in tomato (Solanum lycopersicum L.). African J Agric. Res. 2013; 8(44):5585-5591.

31. Yordanov M. Heterosis in tomato. Monographs Theor. Appl. Genet. 1983; 6:189-219. 